\title{
The Nonlinear Evolution of the Inviscid Secondary Instability of Streamwise Vortex Structures
}

\author{
Nicholas D. Blackaby and Philip Hall * \\ Department of Mathematics, \\ University of Manchester, \\ Manchester, M13 9PL, UK.
}

November 21, 1994

The weakly nonlinear evolution of an inviscid marginally unstable wave growing on a boundary layer supporting a streamwise vortex structure is investigated. The nonlinear growth of the wave is found to be controlled by the diffusion layer located at the edge of the critical layer associated with the wave. The evolution equation is found to depend on the upstream history of the wave and the solution of the equation suggests that the wave either restructures the mean state so as to make it stable or develops a singularity at a finite distance downstream of the point of neutral stability.

*The second author's research was supported by the National Aeronautics and Space Administration under NASA Contract No. NAS1-19480 while the author was in residence at the Institute for Computer Applications in Science and Engineering (ICASE), M/S 132c, NASA Langley Research Center, Hampton, VA, 23681-0001. 


\section{Introduction}

Streamwise vortices are known to be an important ingredient of the transition process in boundary layer flows over both curved and flat walls. In the case of flat walls the streamwise vortices are an initially passive product of wave interactions, see for example Hall and Smith (1991), whilst for curved walls the vortex can be the primary instability of the undisturbed flow. In recent years there has been much interest in the breakdown process of such vortices. In the context of Görtler vortices the experiments of Swearingen and Blackwelder (1987) showed that the initially steady vortex structure caused by wall curvature undergoes a secondary bifurcation to an unsteady three-dimensional flow. This secondary instability can be of either the sinuous or varicose type. In flat plate boundary layers the secondary instability is invariably found to be of the varicose type.

The first attempt to understand the breakdown process for Görtler vortices was given by Hall and Seddougui (1989). In that calculation the small wavelength Görtler vortex flow investigated by Hall and Lakin (1988) was localized in the shear layers trapping the region of vortex activity. Analytical progress for this case is made possible by the assumption of small vortex wavelength and Hall and Seddougui (1989) showed that wavy (i.e. sinuous) modes of instability are possible and are localized near the top and bottom of the vortices. No mode of the varicose type was found to be unstable. If such a mode exists at small vortex wavelengths then it is presumably not trapped in the region where the vortex activity decays to zero.

In order to understand the origin of this secondary instability mechanism, Hall and Horseman (1991) investigated the inviscid linear instability of a Görtler vortex. The instability analysis for such a flow is greatly simplified by the fact that the streamwise velocity component of the flow in the presence of a Görtler vortex remains an order of magnitude larger than the normal and spanwise velocity components. This means that an inviscid disturbance to the vortex flow satisfies a twodimensional form of the Rayleigh equation dependent only on the streamwise velocity component of the vortex. Note here that the lengthscale and timescale of the inviscid mode are relatively short so that nonparallel effects are formally negligible in the leading order inviscid instability analysis. The modified two-dimensional Rayleigh equation found by Hall and Horseman (1991) was simultaneously found in the context of vortex wave interaction theory by Hall and Smith (1991). The numerical solution of the eigenvalue problem associated with the modified Rayleigh equation was discussed by Hall and Horseman (1991) who found that both sinuous and varicose modes become unstable as the vortex develops in the streamwise direction. The question of which mode is the most unstable is a function of the history of the vortex, its wavelength and the Görtler number. However, Hall and Horseman (1991) were able to obtain quantitative agreement with the experimental measurements of Swearingen and Blackwelder (1987). Subsequently the instability problem was examined again by Li and Malik (1994) who found that Hall and Horseman (1991) had missed the most unstable varicose mode. Note that this latter mode is in fact the second most unstable overall and that otherwise the results of Li and Malik (1994) were consistent with those of Hall and Horseman (1991).

Related investigations of the problem discussed above have been given by Liu and Domardzki (1993) and Yu and Liu (1994). In the former paper a Direct Navier-Stokes (DNS) simulation of transition in the Görtler problem was carried out for parallel flows and the results obtained are 
in broad agreement with those of Hall and Horseman (1991). Yu and Liu (1994) reconsidered the instability problem of Hall and Horseman (1991) but retained some viscous terms in their approximation. Yu and Liu (1994) are critical of Hall and Horseman (1991) because of their neglect of viscosity. However, this criticism is perhaps surprising since viscous effects are certainly a second order effect and in any case the equations solved by Yu and Liu (1994) retain only some of the second order effects. In effect the criticism of Yu and Liu (1994) is equivalent arguing that the Orr-Sommerfeld equation rather than the Rayleigh equation should be used to describe inviscid instabilities of parallel flows.

In this paper we shall describe the evolution of the inviscid mode found by Hall and Horseman (1991). This will be done using viscous-critical-layer and diffusion-layer theories in the context of a weakly nonlinear instability theory. In particular, we shall consider the evolution of a mode near the critical streamwise location where the vortex structure has developed sufficiently to (first) render the now three-dimensional boundary-layer flow unstable to inviscid modes (note that, for example, incompressible two-dimensional Blasius-boundary-layer flow does not support inviscid instability). At such a location, the flow is marginally unstable and we can consider the evolution of the most dangerous (important) mode. Related work has been carried out by Wu (1993) and Smith, Brown \& Brown (1993).

Although the analysis we give is for vortex flows generated by wall curvature, it is valid for any flow where one of the velocity components depends on two spatial variables and is larger than the other two components. For such more general flows the periodicity in the spanwise direction which we assume in this paper must be replaced by an appropriate condition in order to derive the required solvability condition. The procedure adopted in the rest of this paper is as follows: In Section 2 we formulate the problem to be considered; in Section 3 we determine the outer solution of the perturbation equations and the required form of the solvability condition, while the critical layer is discussed in Section 4. At the edge of the critical layer a diffusion layer is required in order to account for the mean flow correction; this layer is discussed in Section 5 and the evolution equation is derived; the solution of the evolution equation and some conclusions are given in Section 6.

\section{Formulation}

The nondimensionalised Navier-Stokes equations for an incompressible flow may be written in the form

$$
\begin{gathered}
u_{x}+v_{y}+w_{z}, \\
u_{t}+u u_{x}+v u_{y}+w u_{z}=-p_{x}+\operatorname{Re}^{-1}\left(u_{x x}+u_{y y}+u_{z z}\right), \\
v_{t}+u v_{x}+v v_{y}+w v_{z}=-p_{y}+\operatorname{Re}^{-1}\left(v_{x x}+v_{y y}+v_{z z}\right), \\
w_{t}+u w_{x}+v w_{y}+w w_{z}=-p_{z}+\operatorname{Re}^{-1}\left(w_{x x}+w_{y y}+w_{z z}\right),
\end{gathered}
$$

where $R e$ is the Reynolds number. 
We define new variables $Y, Z$ by writing

$$
y=R e^{-1 / 2} Y, \quad z=R e^{-1 / 2} Z,
$$

and assume a three-dimensional boundary-layer flow (i.e. Blasius flow plus streamwise vortex) of the form:

$$
(u, v, w, p)=\left(\bar{u}(x, Y, Z), \operatorname{Re}^{-1 / 2} \bar{v}(x, Y, Z), \operatorname{Re}^{-1 / 2} \bar{w}(x, Y, Z), p_{\infty}+\operatorname{Re}^{-1} \bar{p}(x, Y, Z)\right) .
$$

This flow can be generated by several mechanisms but the most obvious one is wall curvature. In that case $x, y$ in (2.1) measure distance along and normal to the wall and curvature terms must be inserted into those equations. We then find that the flow is determined by

$$
\begin{gathered}
\bar{u}_{x}+\bar{v}_{Y}+\bar{w}_{Z}, \\
\bar{u} \bar{u}_{x}+\bar{v} \bar{u}_{Y}+\bar{w} \bar{u}_{Z}=\bar{u}_{Y Y}+\bar{u}_{Z Z}, \\
\bar{v}_{t}+\bar{u} \bar{v}_{x}+\bar{v} \bar{v}_{Y}+\bar{w} \bar{v}_{Z}+\frac{1}{2} G \bar{u}^{2}=-\bar{p}_{Y}+\bar{v}_{Y Y}+\bar{v}_{Z Z}, \\
\bar{w}_{t}+\bar{u} \bar{w}_{x}+\bar{v} \bar{w}_{Y}+\bar{w} \bar{w}_{Z}=-\bar{p}_{Z}+\bar{w}_{Y Y}+\bar{w}_{Z Z} ;
\end{gathered}
$$

which must be solved in conjunction with suitable boundary conditions such as 'no-slip' at the wall (solid boundary) and with the solution tending towards a uniform flow at infinity (far from the solid boundary). The parameter $G$ appearing in (2.3) is the Görtler number. The extra curvature terms to be inserted in (2.1) play no role in the nonlinear inviscid instability problem to be investigated here so for convenience we choose not to insert them. Note also that, from Hall and Bennett (1986), the Görtler equations are perhaps more conveniently derived by starting with (2.1) and then making an appropriate Prandtl transformation. Again if this route is followed the extra terms to be inserted into (2.1) play no role in the following analysis. Thus in the present investigation it is sufficient for us to use (2.1) to describe the nonlinear state perturbed around the incoming vortex flow given above. Whether the vortex field is generated by curvature, turbulence screens upstream, or a localized bump at the wall is irrelevant in the following discussion.

In general the solution of (2.3) is a numerical task, see Hall (1988), and it turns out that nonlinear effects stabilize the growth of Görtler vortices; however when a large spanwise wavenumber assumption is made, Hall \& Lakin (1988) demonstrated that much analytical progress can be made towards the solution of these equations.

Let us first recap the linear inviscid stability problem for this three-dimensional boundarylayer flow. In the neighbourhood of a point $x_{0}$ the flow is perturbed by a small inviscid disturbance proportional to

$$
E=\exp [i(\alpha X-\Omega T)]
$$

where

$$
x=x_{0}+R e^{-1 / 2} X \text { and } \quad t=R e^{-1 / 2} T,
$$

and $\alpha, \Omega$ are the (streamwise) wavenumber and frequency of the linear, inviscid secondary instability. The expansions for the velocities and pressure are

$$
(u, v, w, p)=\left(\bar{u}, R e^{-1 / 2} \bar{v}, R e^{-1 / 2} \bar{w}, p_{\infty}+\operatorname{Re}^{-1} \bar{p}(x, Y, Z)\right)+\epsilon((\hat{u}, \hat{v}, \hat{w}, \hat{p}) E+c . c .),
$$


where 'c.c.' represents complex conjugate; barred quantities correspond to the three-dimensional boundary-layer flow; and the disturbance quantities $\hat{u}, \hat{v}, \hat{w}, \hat{p}$ are, in particular, functions of $x, Y$ and $Z$ (but not $X$ or $T$ ).

After a little algebra we find that the pressure perturbation $\hat{p}$ satisfies the modified Rayleigh pressure equation

$$
\frac{\partial}{\partial Y}\left[\frac{\hat{p}_{Y}}{(\bar{u}-c)^{2}}\right]+\frac{\partial}{\partial Z}\left[\frac{\hat{p}_{Z}}{(\bar{u}-c)^{2}}\right]-\frac{\alpha^{2} \hat{p}}{(\bar{u}-c)^{2}}=0,
$$

with boundary conditions

$$
\hat{p}_{Y}(y=0)=0, \quad \hat{p}(Y \rightarrow \infty)=0,
$$

where $c=\Omega / \alpha$. This equation was derived by Hall and Smith (1991) who were concerned with vortex-wave interactions and by Hall and Horseman (1991) in the context of secondary instabilities of Görtler vortices. The eigenvalue problem for $c \equiv c(\bar{u}, x, \alpha)$ (temporal stability problem) associated with the partial differential system for $\hat{p}$ was first solved by Hall and Horseman (1991). Here we shall consider the more appropriate spatial instability problem in the presence of nonlinear effects.

Note that $c$ is not a function of $Z$; if we consider neutral disturbances (those having $c$ entirely real), equation (2.6a) is singular at $Y=Y_{C} \equiv f(x, Z)$, (say) where $\bar{u}=c$. Thus for threedimensional boundary-layers, the critical layer is 'wavy' in the sense that the location of the critical level (where the equation is singular) is a function of spanwise location $Z$. Note further that the neutral value of $c$, and hence $Y_{C} \equiv f(x, Z)$, are not known in advance of a numerical solution of the eigenvalue-problem $(2.6 \mathrm{a}-\mathrm{c})$.

In Figure 1 we show some results for the most dangerous odd mode of instability for a Görtler vortex flow. The shape of the curve is similar to that which would be obtained for an inflectional uni-directional velocity field by solving the standard Rayleigh equation. In Figure 2 we show the normal and streamwise perturbation velocity components for an unstable mode. Note that the mode is concentrated towards the edge of the boundary layer. Since the mode is not neutral it does not exhibit a critical layer behaviour. In fact it is a difficult numerical procedure to solve (2.6) in the neutral case and results are not yet available. Now let us consider the nonlinear problem and as a starting point we discuss the outer region away from the critical layer.

\section{The outer solution for weakly nonlinear inviscid modes}

In order to derive the desired nonlinear evolution equation for the amplitude of an inviscid disturbance mode, it is necessary to split the three-dimensional boundary-layer flow into distinct regions (layers), each corresponding to different dominant physical effects locally governing the inviscid disturbance; see Figure 3. Let us first consider the flow solutions in regions Ia, b, away from the critical layer, diffusion layer and solid boundaries (the wall) i.e. the bulk of the boundarylayer. Our aim is to derive the solvability condition associated with an inhomogeneous form of (2.6a-c). This solvability condition, together with expressions for certain 'jumps' in flow velocities in crossing the critical layer, (to be derived in the later subsections) will yield an evolution equation for the spatial evolution of the disturbance amplitude. 
Before proceeding any further, let us consider the various streamwise lengthscales which are present in the analysis. In fact, we employ multiple scales in the streamwise direction $x$ :

$$
x=x_{0}+\mu \tilde{x}, \quad x=x_{0}+R e^{-1 / 2} \mu^{-1} \tilde{X}, x=x_{0}+R e^{-1 / 2} X .
$$

Here $x_{0}$ now denotes the neutral $x$-station (in fact, $x_{0}$ is a point of marginal instability as the flow first becomes unstable to linear inviscid disturbances here); $\mu \tilde{x}$ is a small change in $x$-location from $x_{0}$ (assuming $\mu \tilde{x}>0$, then the flow is now slightly unstable: hence the weakly nonlinear analysis to follow); $R e^{-1 / 2} \mu^{-1} \tilde{X}$ is the wave-amplitude modulation scale; and $R e^{-1 / 2} X$ is the scale on which the waves oscillate. Note that we are considering the purely spatial stability problem and thus there are no slow time effects.

We consider 'fixed-frequency' disturbances so that $\Omega$ is fixed; thus the non-neutrality is entirely due to the the change in $x$-location $\mu \tilde{x}$. Note that the developing three-dimensional boundarylayer, and hence the neutral streamwise-wavenumber $\alpha$, are functions of $x$. In addition it should be noted that, if we were considering a three-dimensional shear-layer rather than a three-dimensional boundary-layer, we would not consider $\Omega$ fixed. The non-neutrality would then be entirely due to the $\Omega$ perturbation (i.e. a Strouhal number perturbation).

Since the normal and spanwise velocity components of the unperturbed flow are relatively small only the streamwise component $\bar{u}$ of the three-dimensional boundary-layer flow enters the problem to orders of concern. We write

$$
\bar{u}=\bar{u}_{0}+\mu \bar{u}_{1}+\ldots, \quad \bar{u}_{0}(Y, Z)=\bar{u}\left(x_{0}, Y, Z\right), \quad \bar{u}_{1}(Y, Z)=\bar{u}_{0 x}\left(x_{0}, Y, Z\right) \tilde{x}, \cdots .
$$

Further, we only need to consider two terms of the fundamental (proportional to $E^{ \pm}$) and write

$$
(\hat{u}, \hat{v}, \hat{w}, \hat{p})=\left(\hat{u}_{1}, \hat{v}_{1}, \hat{w}_{1}, \hat{p}_{1}\right)+\cdots+\mu\left(\hat{u}_{2}, \hat{v}_{2}, \hat{w}_{2}, \hat{p}_{2}\right)+\cdots
$$

(i) The leading fundamental term

The leading order problem for $\left(\hat{u}_{1}, \hat{v}_{1}, \hat{w}_{1}, \hat{p}_{1}\right)$ is a partial differential system in $Y, Z$ and its solution may be written in the form

$$
\left(\hat{u}_{1}, \hat{v}_{1}, \hat{w}_{1}, \hat{p}_{1}\right)=A(\tilde{X})\left(\hat{u}_{1}^{(1)}, \hat{v}_{1}^{(1)}, \hat{w}_{1}^{(1)}, \hat{p}_{1}^{(1)}\right) E+\text { c.c. }
$$

where $A$ is an amplitude function and $\hat{u}_{1}^{(1)}, \hat{v}_{1}^{(1)}, \hat{w}_{1}^{(1)}$ and $\hat{p}_{1}^{(1)}$ satisfy

$$
\begin{gathered}
i \alpha \hat{u}_{1}^{(1)}+\hat{v}_{1 Y}^{(1)}+\hat{w}_{1 Z}^{(1)}=0, \quad i \alpha\left(\bar{u}_{0}-c\right) \hat{u}_{1}^{(1)}+\bar{u}_{0 Y} \hat{v}_{1}^{(1)}+\bar{u}_{0 Z} \hat{w}_{1}^{(1)}=-i \alpha \hat{p}_{1}^{(1)} \\
i \alpha\left(\bar{u}_{0}-c\right) \hat{v}_{1}^{(1)}=-\hat{p}_{1 Y}^{(1)}, \quad i \alpha\left(\bar{u}_{0}-c\right) \hat{w}_{1}^{(1)}=-\hat{p}_{1 Z}^{(1)} .
\end{gathered}
$$

These lead to the eigenvalue problem $(2.6)$ (with $\hat{p}_{1}^{(1)}$ replacing $\hat{p}$ ) to determine the neutral solution at $x=x_{0}$. We fix $\hat{p}_{1}^{(1)}$ by the normalization:

$$
\hat{p}_{1}^{(1)}=\hat{p}_{\infty}(Z) e^{-\alpha Y} \quad \text { as } \quad Y \rightarrow \infty
$$


where $\hat{p}_{\infty}(Z)$ is prescribed at some value of $Z$.

Following Hall and Smith (1991) let us now consider the behaviours of $\hat{u}_{1}^{(1)}, \ldots, \hat{p}_{1}^{(1)}$ near the critical layer at $Y=Y_{c} \equiv f(Z)$. Defining $s=Y-Y_{c}$ (so that $\partial_{Z} \rightarrow \partial_{Z}-f_{Z} \partial_{s}$ ), we write

$$
\begin{gathered}
\bar{u}_{0}=c+\lambda(x, Z) s+\lambda_{2}(x, Z) s^{2} / 2+\lambda_{3}(x, Z) s^{2} / 6+\cdots, \\
\bar{u}_{1}=\bar{u}_{10}(x, Z)+\bar{u}_{11}(x, Z) s+\bar{u}_{12}(x, Z) s^{2} / 2+\cdots .
\end{gathered}
$$

The method of Frobenius gives $\hat{p}_{1}^{(1)}$ in the form

$$
\hat{p}_{1}^{(1)}=\phi_{1}+b_{1 \pm}(Z) \phi_{2},
$$

where

$$
\phi_{1}=\phi_{10}(Z)+\phi_{11}(Z) . s+\phi_{12}(Z) . s^{2}+\phi_{13 L}(Z) . s^{3} \ln s+0 . s^{3}+\cdots, \quad \phi_{2}=s^{3}+\cdots .
$$

Note that $\phi_{10}(Z)$ and $b_{1 \pm}$ have to be determined numerically (the \pm corresponding to above and below critical level respectively); while, in particular,

$$
\begin{gathered}
\Delta \phi_{11}=f_{Z} \phi_{10 Z}, \\
2 \lambda \Delta^{2} \phi_{12}=\lambda \Delta \phi_{10 Z Z}-\left(2 \lambda_{Z}+\lambda f_{Z} f_{Z Z}\right) \phi_{10 Z}-\alpha^{2} \lambda \Delta \phi_{10}
\end{gathered}
$$

and

$$
\begin{aligned}
6 \lambda \Delta \phi_{13 L}= & 4 \lambda f_{Z} \phi_{12 Z}+2\left(3 \lambda_{2} \Delta-4 f_{Z} \lambda_{Z}+2 \lambda f_{Z Z}\right) \phi_{12}-2 \lambda \phi_{11 Z Z}+2\left(2 \lambda_{Z}-\lambda_{2} f_{Z}\right) \phi_{11 Z} \\
& +\left(2 \alpha^{2} \lambda-2 f_{Z} \lambda_{2 Z}+\lambda_{2} f_{Z Z}\right) \phi_{11}-\lambda_{2} \phi_{10 Z Z}+2 \lambda_{2 Z} \phi_{10 Z}+\alpha^{2} \lambda_{2} \phi_{10},
\end{aligned}
$$

where we have defined

$$
\Delta=1+f_{Z}^{2}
$$

Here, following Hall and Smith (1991), we take $\phi_{13 L}=0$. If it were non-zero, at $O\left(R e^{-1 / 6}\right)$ a jump in $\hat{v}$ across the sole critical layer would be induced and as we are not looking at upperbranch scalings there would be no wall layer effects to counteract this jump; hence this jump and the coefficient of the logarithm-term must be zero.

The velocities $\hat{u}_{1}^{(1)}, \hat{v}_{1}^{(1)}$ and $\hat{w}_{1}^{(1)}$ have the following properties near the critical layer:

$$
\hat{u}_{1}^{(1)}=d_{1 Z} s^{-1}+\cdots, \quad \hat{v}_{P 1}^{(1)} \equiv \hat{v}_{1}^{(1)}-f_{Z} \hat{w}_{1}^{(1)}=d_{2}+\cdots, \quad \hat{w}_{1}^{(1)}=-i \alpha d_{1} s^{-1}+\cdots,
$$

where

$$
d_{1}=-\phi_{10 Z} / \alpha^{2} \lambda \Delta \quad \text { and } \quad d_{2}=\left(-2 \Delta \phi_{12}+f_{Z} \phi_{11 Z}\right) / i \alpha \lambda . \quad(3.8 a-e)
$$


(ii) The largest forced fundamental

Let us now consider the largest forced term of the fundamental (due to non-neutrality effects), denoted by $\left(\hat{u}_{2}^{(1)}, \hat{v}_{2}^{(1)}, \hat{w}_{2}^{(1)}, \hat{p}_{2}^{(1)}\right)$. It is found that

$$
\frac{\partial}{\partial Y}\left[\frac{\hat{p}_{2 Y}^{(1)}}{(\bar{u}-c)^{2}}\right]+\frac{\partial}{\partial Z}\left[\frac{\hat{p}_{2 Z}^{(1)}}{(\bar{u}-c)^{2}}\right]-\frac{\alpha^{2} \hat{p}_{2}^{(1)}}{(\bar{u}-c)^{2}}=\frac{R_{2}}{(\bar{u}-c)^{2}}
$$

with boundary conditions

$$
\hat{p}_{2 Y}^{(1)}(Y=0)=0, \quad \hat{p}_{2}^{(1)}(Y \rightarrow \infty)=0
$$

and

$$
R_{2}=R_{2}^{(1)} \frac{\partial A}{\partial \tilde{X}}+R_{2}^{(0)} A
$$

where

$$
\begin{gathered}
R_{2}^{(1)}=\left[\frac{c i}{\alpha\left(\bar{u}_{0}-c\right)}\left(\hat{p}_{1 Y Y}^{(1)}+\hat{p}_{1 Z Z}^{(1)}-\alpha^{2} \hat{p}_{1}^{(1)}\right)-2 i \alpha \hat{p}_{1}^{(1)}\right], \\
R_{2}^{(0)}=-\left[\frac{\bar{u}_{1}}{\left(\bar{u}_{0}-c\right)}\left(\hat{p}_{1 Y Y}^{(1)}+\hat{p}_{1 Z Z}^{(1)}-\alpha^{2} \hat{p}_{1}^{(1)}\right)-\frac{2}{\left(\bar{u}_{0}-c\right)}\left(\bar{u}_{1 Y} \hat{p}_{1 Y}^{(1)}+\bar{u}_{1 Z} \hat{p}_{1 Z}^{(1)}\right)\right] .
\end{gathered}
$$

Note that the equation for $\hat{p}_{2}^{(1)}$ is an inhomogeneous form of that for $\hat{p}_{1}^{(1)}$; as mentioned previously, we must determine a solvability condition for this equation, to ensure that it has a solution. This solvability condition will (indirectly) lead to the desired evolution equation. To derive the solvability condition we will essentially follow the conventional method of multiplying the inhomogeneous equation by the adjoint of the homogeneous equation and then integrate over the range $0 \leq Y \leq \infty, 0 \leq Z \leq 2 \pi$ (the latter corresponding to a complete period). Note that we are assuming that the disturbance has the same period as the basic flow. The latter assumption is justified by the fact that Hall and Horseman (1991) were unable to find any subharmonic disturbances of the modified Rayleigh pressure equation. As in all critical layer problems, special care must be taken to deal with the singular nature of the equations (i.e. the critical layers); however extra care is necessary for the current problem due to the 'waviness' ( $Z$-dependence of $Y_{c} \equiv f(Z)$ ) of the critical layer. The fact that additional care is required can be explained as follows: note that in $(Y, Z)$-coordinates (i) for any fixed $Z$-value there is a unique critical- $Y$ value, but (ii), for a fixed $Y$-value there is either no critical $Z$-values or many.

Thus we look for co-ordinates which describe the critical-level more suitably; note that the Prandtl-transformation $(s=Y-f(Z))$ does 'level' out the critical-layer but, at the same time, leaves the solid boundary 'wavy' (i.e. it is described by $Y=-f(Z)$ ). Instead we introduce the normal variable

$$
\xi=Y / f(Z)
$$

this transformation 'flattens' out the critical-level (it corresponds to $\xi=1$ ) and also leaves the wall flat $(\xi=0)$. Note that this transformation involves $f(Z)$ and thus could not have been used from the outset i.e. it involves information from the solution of the homogeneous problem (1.6a-c). 
Note that

$$
\partial / \partial_{Y} \rightarrow(1 / f(Z)) \partial / \partial_{\xi} ; \quad \partial / \partial_{Z} \rightarrow \partial / \partial_{Z}+g(Z) \xi \partial / \partial_{\xi},
$$

where $g=-f^{\prime} / f$. In these co-ordinates, the homogeneous equation takes the form:

$$
\begin{gathered}
\left(1 / f^{2}\right) \frac{\partial}{\partial \xi}\left[\frac{\hat{p}_{1 \xi}^{(1)}}{(\bar{u}-c)^{2}}\right]+\left(\partial / \partial_{Z}+g \xi \partial / \partial_{\xi}\right)\left[\frac{\left(\partial / \partial_{Z}+g \xi \partial / \partial_{\xi}\right) \hat{p}_{1}^{(1)}}{(\bar{u}-c)^{2}}\right]-\frac{\alpha^{2} \hat{p}_{1}^{(1)}}{(\bar{u}-c)^{2}}=0, \\
\hat{p}_{1 \xi}^{(1)}(\xi=0)=0, \quad \hat{p}_{1}^{(1)}(\xi=\infty)=0 ;
\end{gathered}
$$

whilst the adjoint, $q$ say, satisfies

$$
\begin{gathered}
\left(1 / f^{2}\right) \frac{\partial}{\partial \xi}\left[\frac{q_{\xi}}{(\bar{u}-c)^{2}}\right]+\left(\partial / \partial_{Z}-g \partial / \partial_{\xi} \xi\right)\left[\frac{\left(\partial / \partial_{Z}-g \partial / \partial_{\xi} \xi\right) q}{(\bar{u}-c)^{2}}\right]-\frac{\alpha^{2} q}{(\bar{u}-c)^{2}}=0 \\
q_{\xi}(\xi=0)=0, \quad q(\xi=\infty)=0 .
\end{gathered}
$$

Therefore in these transformed co-ordinates the pressure equation is not self-adjoint.

Thus to derive the solvability condition for $(3.9 \mathrm{a}-\mathrm{f})$ we first transform to $(\xi, Z)$ variables; multiply both sides by the adjoint $q$ (defined above) and then integrate both sides over the range $0 \leq \xi \leq \infty, 0 \leq Z \leq 2 \pi$, excluding the critical layer. This gives

$$
\begin{gathered}
\int_{0}^{2 \pi} d Z\left[\frac{1}{(\bar{u}-c)^{2}}\left(\xi g\left(q \hat{p}_{2 Z}^{(1)}-\hat{p}_{2}^{(1)} q_{Z}\right)+\frac{1}{f^{2}}\left(q \hat{p}_{2 \xi}^{(1)}-\hat{p}_{2}^{(1)} q_{\xi}\right)+g^{2}\left(\xi^{2} q \hat{p}_{2 \xi}^{(1)}-\xi \hat{p}_{2}^{(1)}(\xi q) \xi\right)\right]_{\xi=1^{-}}^{\xi=1^{+}}=\right. \\
-\int_{0}^{2 \pi} f_{0}^{\infty} \frac{q R_{2}}{(\bar{u}-c)^{2}} d \xi d Z,
\end{gathered}
$$

where the bar through the integral represents the finite part (i.e. excluding critical layer effects).

Near the critical level, $q$ is of the form

$$
q=q_{10}+q_{11}(\xi-1)+q_{12}(\xi-1)^{2}+0(\xi-1)^{3} \ln (\xi-1)+q_{13}(\xi-1)^{3}+\cdots,
$$

whilst $R_{2}^{(0)}, R_{2}^{(1)}$ have the forms

$$
R_{2}^{(k)}=r_{-1}^{(k)}(\xi-1)^{-1}+r_{0}^{(k)}+r_{1}^{(k)}(\xi-1)+r_{2}^{(k)}(\xi-1)^{2}+\cdots, k=0,1,
$$

(the expressions for the coefficients are simply obtained by expanding $(3.9 \mathrm{e}, \mathrm{f})$ near the critical layer, however they are somewhat long and for the sake of brevity are not given here) while $\hat{p}_{2}^{(1)}$ has the form

$$
\hat{p}_{2}^{(1)}=a_{2} \phi_{1}+b_{2 \pm} \phi_{2}+\hat{p}_{2 P I}^{(1)} .
$$

Here the first two terms correspond to solutions of the homogeneous equation, $a_{2}, b_{2 \pm}$ are as yet undetermined constants, and subscript $P I$ denotes particular integral. We define $a_{2}, b_{2 \pm}$ so that $\hat{p}_{2 P I}^{(1)}$ has no terms in $(\xi-1)^{0},(\xi-1)^{3}$; i.e. $\hat{p}_{2 P I}^{(1)}$ is properly defined. Then, near the critical layer, $\hat{p}_{2 P I}^{(1)}$ has the form

$$
\hat{p}_{2 P I}^{(1)}=\beta_{1}(\xi-1)+\beta_{2}(\xi-1)^{2}+\beta_{3 L}(\xi-1)^{3} \ln (\xi-1)+0(\xi-1)^{3}+\cdots,
$$


where

$$
\beta_{3 L}=\beta_{3 L}^{(1)} \frac{\partial A}{\partial \tilde{X}}+\beta_{3 L}^{(0)} A
$$

and

$$
\beta_{3 L}^{(k)}=\frac{1}{\lambda^{2}}\left(q_{12} r_{-1}^{(k)}+q_{11} r_{0}^{(k)}+q_{10} r_{1}^{(k)}\right)-\frac{1}{\lambda^{3}}\left(q_{11} r_{-1}^{(k)} \lambda_{2}+q_{10} r_{0}^{(k)} \lambda_{2}\right)+\frac{q_{10} r_{-1}^{(k)}}{2 \lambda^{2}}\left(\frac{3 \lambda_{2}^{2}}{2 \lambda^{2}}-\frac{2 \lambda_{3}}{3 \lambda}\right), k=0,1 .
$$

Thus the solvability condition becomes

$$
\int_{0}^{2 \pi} \frac{3 \Delta q_{10}}{f^{2} \lambda^{2}}\left(b_{2+}-b_{2-}\right) d Z=-\int_{0}^{2 \pi} f_{0}^{\infty} \frac{q R_{2}}{(\bar{u}-c)^{2}} d \xi d Z
$$

Near the critical layer, the Prandtl-transformed normal velocity has the form

$$
\hat{v}_{2 P}^{(1)} \equiv \hat{v}_{2}^{(1)}-f_{Z} \hat{w}_{2}^{(1)}=\pi_{-1}(\xi-1)^{-1}+\pi_{0}+\pi_{1 L}(\xi-1) \ln (\xi-1)+\pi_{1 \pm}(\xi-1)+\cdots \quad(\xi>1)
$$

where

$$
\pi_{1 L}=-\frac{3 \Delta}{i \alpha \lambda} \beta_{3 L} \quad \text { and } \quad \pi_{1+}-\pi_{1-}=-\frac{3 \Delta}{i \alpha \lambda}\left(b_{2+}-b_{2-}\right) .
$$

Note that for $\xi<1$, the logarithm $\ln (\xi-1)$ in (3.16a) must be replaced by $\ln |\xi-1|-i \pi$; the negative sign in front of $i \pi$ follows from an inspection of the Stokes lines of the operator that occurs in the governing equations for the linear problem in the critical-layer.

Thus, the linear part of the jump that $\hat{v}_{2 P \xi}^{(1)}$ suffers across the critical layer is

$$
\left[\hat{v}_{2 P \xi}^{(1)}\right]_{1-}^{1+}=\pi_{1+}-\pi_{1-}+i \pi \pi_{1 L} ;
$$

an expression for the nonlinear contribution to the jump is calculated in the following two subsections before the two expressions are combined to give the evolution equation.

\section{The Critical Layer}

Let us now consider the critical-layer flow (corresponding to region III in Figure 3) with our aim to calculate expressions for the jumps in the solvability condition obtained in the previous subsection; will shall find that another region, the so-called diffusion layer, also needs to be considered and this is the subject of the next subsection. The analysis has similarities to that given in Appendix B of Hall \& Smith (1991); Appendix A of Brown, Brown, Smith \& Timoshin (1993); and Wu (1993). Therefore only the essential details of the analysis will be given here.

The critical layer is centred on $Y=Y_{c} \equiv f(x, Z)$ and has thickness $O\left(R e^{-1 / 6}\right)$ relative to the boundary-layer thickness; it is a viscous critical layer. We introduce the critical layer normal variable $\eta \sim O(1)$ where

$$
R e^{1 / 2} y=Y=f(Z)+R e^{-1 / 6} \eta,
$$


so that now

$$
\frac{\partial}{\partial Z} \rightarrow \frac{\partial}{\partial Z}-R e^{1 / 6} f_{Z} \frac{\partial}{\partial \eta}
$$

and the transformed normal velocity $v_{P}$ is defined by

$$
v_{P}=v-f_{Z} w
$$

The underlying three-dimensional flow has the form

$$
\begin{aligned}
\bar{u}=c+R e^{-1 / 6} \lambda(Z) \eta+R e^{-1 / 3} \lambda_{2}(Z) \eta^{2} / 2+\cdots+ \\
\mu \bar{u}_{10}+\mu R e^{-1 / 6} \bar{u}_{11}+\mu R e^{-1 / 3} \bar{u}_{12} / 2+\cdots
\end{aligned}
$$

Since we are looking at a viscous critical layer (the flow is marginally unstable) we must assume that,

$$
\mu \ll R e^{-1 / 6}
$$

However, depending on the size of $\mu$, the above expansion (4.2) will be disordered.

In the critical layer the perturbation to the three-dimensional underlying base flow has the following form:

$$
\begin{aligned}
\left(u, v_{P}, w, p\right)=\epsilon & {\left[\left(\operatorname{Re}^{1 / 6} \hat{U}_{1}, \hat{V}_{P 1}, R e^{1 / 6} \hat{W}_{1}, \hat{P}_{1}\right) E+c . c .\right] } \\
& +\cdots+\epsilon^{2}\left(\operatorname{Re}^{1 / 2} \hat{U}_{0}, R e^{1 / 3} \hat{V}_{P 0}, R e^{1 / 2} \hat{W}_{0}, R e^{1 / 3} \hat{P}_{0}\right) E^{0}+\cdots .
\end{aligned}
$$

Here only the terms of direct interest have been shown. The second harmonic does not contribute to the nonlinear jump and therefore has not been included above.

\section{(i) The fundamental}

Writing

$$
\hat{U}_{1}=\hat{U}_{1}^{(1)}+R e^{-1 / 6} \hat{U}_{1}^{(2)}+\cdots,
$$

with similar expansions for $\hat{V}_{P 1}, \hat{W}_{1}$ and $\hat{P}_{1}$, and substituting into the governing (Prandtl-transformed) equations leads to the following system for the leading order fundamental

$$
\begin{gathered}
i \alpha \hat{U}_{1}^{(1)}+\hat{V}_{P 1 \eta}^{(1)}+\hat{W}_{1 Z}^{(1)}=0, \\
i \alpha \lambda \eta \hat{U}_{1}^{(1)}+\lambda \hat{V}_{P 1}^{(1)}+\lambda_{Z} \eta \hat{W}_{1}^{(1)}=-i \alpha \hat{P}_{1}^{(1)}+\Delta \hat{U}_{1 \eta \eta}^{(1)}, \\
\hat{P}_{1 \eta}^{(1)}=0 \\
i \alpha \lambda \eta \hat{W}_{1}^{(1)}=-\hat{P}_{1 Z}^{(1)}+f_{Z} \hat{P}_{1 \eta}^{(2)}+\Delta \hat{W}_{1 \eta \eta}^{(1)},
\end{gathered}
$$

which must be solved subject to the condition that solutions match to their outer counterparts as $\eta \rightarrow \pm \infty$. The $y$-momentum equation of the next order problem (for $\hat{U}_{1}^{(2)}$, etc) yields, in particular,

$$
\hat{P}_{1 \eta}^{(2)}=f_{Z} \hat{P}_{1 Z}^{(1)} / \Delta
$$


enabling a solution of (4.5a-d) to be determined. The solutions which match with the outer flow can be found in the paper by Brown, Brown, Smith \& Timoshin (1993); in our notation they are

$$
\begin{gathered}
\hat{P}_{1}^{(1)}=A(\tilde{X}) \phi_{10}(Z), \\
\hat{W}_{1}^{(1)}=-\frac{1}{a^{2 / 3} \Delta^{2}} \frac{\partial \hat{P}_{1}^{(1)}}{\partial Z} \int_{0}^{\infty} \exp \left[-i a^{1 / 3} \eta t-t^{3} / 3\right] \mathrm{d} t \\
\hat{U}_{1}^{(1)}=\frac{i}{\alpha} \frac{\partial \hat{W}_{1}^{(1)}}{\partial Z}-\frac{i}{4 \alpha}\left(\frac{\lambda_{Z}}{\lambda}+\frac{a_{Z}}{a}\right) \frac{\partial}{\partial \eta}\left(\eta \hat{W}_{1}^{(1)}\right),
\end{gathered}
$$

and

$$
\hat{V}_{P 1}^{(1)}=-\left(\frac{\lambda_{Z}}{\lambda}+\frac{a_{Z}}{a}\right) \frac{\eta \hat{W}_{1}^{(1)}}{4}+d_{2}-\frac{1}{2 i \alpha \lambda \Delta}\left(\frac{\lambda_{Z}}{\lambda}-\frac{f_{Z} f_{Z Z}}{\Delta}\right),
$$

where

$$
a=\lambda \alpha / \Delta \text {. }
$$

At this stage we also point out that $\lambda, \alpha, a, \Delta$ are all real.

It is also possible to consider further terms in the fundamental; in fact, by considering the terms due to the non-neutrality it is possible to derive the logarithmic jump effect deduced earlier from the asymptotic form of the outer solution as the critical layer is approached. As a number of different orders have to be considered, and the answers are algebraically messy, we do not present that analysis here.

\section{(ii) The zeroth harmonic}

We now consider the largest zeroth harmonic, or mean-flow correction, (proportional to $E^{0}$ ) due to the nonlinear interactions of the fundamental. Writing

$$
\hat{U}_{0}=\hat{U}_{0}^{(1)}+R e^{-1 / 6} \hat{U}_{0}^{(2)}+\cdots,
$$

with similar expansions for $\hat{V}_{P 0}, \hat{W}_{0}$ and $\hat{P}_{0}$, and substituting into the governing (Prandtl-transformed) equations leads to the following system for the leading order zeroth harmonic

$$
\begin{gathered}
\hat{V}_{P 0 \eta}^{(1)}+\hat{W}_{0 Z}^{(1)}=0, \\
\Delta \hat{U}_{0 \eta \eta}^{(1)}-\lambda \hat{V}_{P 0}^{(1)}-\lambda_{Z} \eta \hat{W}_{0}^{(1)}=S_{1}, \\
\hat{P}_{0 \eta}^{(1)}=0, \\
f_{Z} \hat{P}_{0 Z}^{(1)}-\Delta \hat{P}_{0 \eta}^{(2)}-2 f_{Z Z} \hat{W}_{1}^{(1)} \hat{W}_{1}^{(1) *}=0, \\
\Delta \hat{W}_{0 \eta \eta}^{(1)}+f_{Z} \hat{P}_{0 \eta}^{(2)}-\hat{P}_{0 Z}^{(1)}=S_{2},
\end{gathered}
$$

where, in particular,

$$
S_{2}=i \alpha \hat{U}_{1}^{(1) *} \hat{W}_{1}^{(1)}+\hat{V}_{P 1}^{(1) *} \hat{W}_{1 \eta}^{(1)}+\hat{W}_{1}^{(1) *} \hat{W}_{1 Z}^{(1)}+\text { c.c.. } \quad(4.9 a-f)
$$


As usual in such studies we choose $\hat{P}_{0}^{(1)}=0$ (any non-zero choice would just correspond to a different mean-flow). Thus

$$
\Delta \hat{W}_{0 \eta \eta}^{(1)}=S_{2}+2 \frac{f_{Z} f_{Z Z}}{\Delta} \hat{W}_{1}^{(1)} \hat{W}_{1}^{(1) *}
$$

from which it follows that

$$
\hat{W}_{0}^{(1)} \rightarrow\left[ \pm F_{w}+C\right] \eta, \quad \hat{V}_{P 0}^{(1)} \rightarrow-\left[ \pm F_{w}+C\right]_{Z} \eta^{2} / 2
$$

and

$$
\hat{U}_{0}^{(1)} \rightarrow \frac{1}{24 \Delta \lambda}\left(\lambda^{2}\left[ \pm F_{w}+C\right]\right)_{Z} \eta^{4}, \quad \text { as } \quad \eta \rightarrow \infty
$$

where

$$
F_{w}(\tilde{X}, Z)=\left(\left(\left|\phi_{10}\right|^{2}\right)_{Z}-\left(\frac{5 a_{Z}}{3 a}+\frac{7 \Delta_{Z}}{2 \Delta}\right)\left|\phi_{10}\right|^{2}\right) \frac{\pi(2 / 3)^{2 / 3} \Gamma(1 / 3)}{a^{5 / 3} \Delta^{5}}|A(\tilde{X})|^{2}, \quad(4.10 a-d)
$$

and it is important at this stage to note that the $\tilde{X}$ and $Z$ dependences of $F_{w}$ are separable.

These asymptotes imply that the zeroth harmonic grows on leaving the critical-layer and that it is necessary to consider another flow region (the diffusion layer) where diffusion effects can counteract this growth. In general such diffusion-layers need to be considered in all nonlinear analyses dealing with viscous critical layers (see, for example, Brown \& Stewartson, 1978; Wu, 1993; cf. the buffer-layer of weakly nonlinear vortex-wave theories).

\section{The Diffusion Layer and the Evolution Equation}

This layer (regions IIa,b in Figure 3) is introduced to take care of the growing mean-flow corrections as the critical layer is left; it turns out that the nonlinear term in the desired evolution equation for $A$ stems entirely from this region. It is necessary to introduce the new scaled normal coordinate

$$
\tilde{\eta}=(Y-f(Z)) /\left(\delta_{2} \Delta^{1 / 2} c^{-1 / 2}\right), \quad \delta_{2}=R e^{-1 / 4} \mu^{-1 / 2} \ll 1 ;
$$

and so here the mean flow has the form

$$
\bar{u}=c+(\Delta / c)^{1 / 2} \delta_{2} \lambda \tilde{\eta}+\cdots
$$

The perturbation of this mean flow has the form

$$
\begin{aligned}
& \left(u, v_{P}, w, p\right)=\epsilon\left[\left(\delta_{2}^{-1} \tilde{U}_{1}, \tilde{V}_{P 1}, \delta_{2}^{-1} \tilde{W}_{1}, \tilde{P}_{1}\right) E+c . c .\right]+\cdots+ \\
& \epsilon^{2}\left(\delta_{2}^{4} R e^{7 / 6} \tilde{U}_{0}, \delta_{2}^{2} R e^{2 / 3} \tilde{V}_{P 0}, \delta_{2} R e^{2 / 3} \tilde{W}_{0}, \delta_{2}^{-1} \tilde{P}_{0}\right) E^{0}+\cdots
\end{aligned}
$$

The fundamental has the expansion

$$
\tilde{U}_{1}=\tilde{U}_{1}^{(1)}+\cdots+\delta_{3} \tilde{U}_{1}^{(2)}+\cdots, \quad \delta_{3}=\epsilon^{2} \delta_{2}^{3} R e^{7 / 6},
$$


with similar series for $\tilde{V}_{P 0}, \tilde{W}_{0}$ and $\tilde{P}_{0}$. Note that the largest fundamental in this region is merely a continuation of the outer solution in the locality of the critical layer:

$$
\left(\tilde{U}_{1}^{(1)}, \tilde{V}_{P 1}^{(1)}, \tilde{W}_{1}^{(1)}, \tilde{P}_{1}^{(1)}\right)=\left(c^{1 / 2} \Delta^{-1 / 2} d_{1 Z} \tilde{\eta}^{-1}, \quad d_{2}, \quad-i \alpha c^{1 / 2} \Delta^{-1 / 2} d_{1} \tilde{\eta}^{-1}, \phi_{10}\right) A(\tilde{X}) .
$$

(i) The leading zeroth harmonic

Writing

$$
\left(\tilde{U}_{0}, \tilde{V}_{P 0}, \tilde{W}_{0}\right)=\left(\tilde{U}_{0}^{(1)}, \tilde{V}_{P 0}^{(1)}, \tilde{W}_{0}^{(1)}\right)+\cdots,
$$

we find that the governing equations for the leading zeroth harmonic are

$$
\begin{gathered}
\Delta^{-1 / 2} c^{1 / 2} \tilde{V}_{P 0 \tilde{\eta}}^{(1)}+\tilde{W}_{0 Z}^{(1)}=0 \\
\left(\frac{\partial}{\partial \tilde{X}}-\frac{\partial^{2}}{\partial \tilde{\eta}^{2}}\right) \tilde{U}_{0}^{(1)}+\frac{\lambda}{c} \tilde{V}_{P 0}^{(1)}+\frac{\Delta^{1 / 2} \lambda_{Z}}{c^{3 / 2}} \tilde{\eta} \tilde{W}_{0}^{(1)}=0
\end{gathered}
$$

and

$$
\left(\frac{\partial}{\partial \tilde{X}}-\frac{\partial^{2}}{\partial \tilde{\eta}^{2}}\right) \tilde{W}_{0}^{(1)}=0
$$

It follows, from matching with the critical-layer solutions, that the boundary conditions at $\tilde{\eta}= \pm 0$ are

$$
\frac{\partial^{4} \tilde{U}_{0}^{(1)}}{\partial \tilde{\eta}^{4}}=\frac{1}{\lambda c}\left(\lambda^{2} F_{ \pm}\right)_{Z}, \quad \frac{\partial^{2} \tilde{V}_{P 0}^{(1)}}{\partial \tilde{\eta}^{2}}=-F_{ \pm Z}, \quad \frac{\partial \tilde{W}_{0}^{(1)}}{\partial \tilde{\eta}}=\Delta^{-1 / 2} c^{1 / 2} F_{ \pm}
$$

where $F_{ \pm}=\Delta\left( \pm F_{w}+C\right) / c$. These equations can be solved using a Fourier transform method to give

$$
\begin{gathered}
\tilde{U}_{0 \tilde{\eta} \tilde{\eta}}^{(1)}= \pm\left(1 / 2 c \pi^{1 / 2}\right) \tilde{\eta} \int_{0}^{\infty} \mathrm{d} t t^{-1 / 2} \exp \left(-\tilde{\eta}^{2} / 4 t\right)\left(\lambda\left[F_{ \pm}(\tilde{X}-t)\right]_{Z}\right. \\
\left.\quad+(1 / 4) \tilde{\eta}^{2} t^{-1} \lambda_{Z} F_{ \pm}(\tilde{X}-t)-(3 / 2) \lambda_{Z} F_{ \pm}(\tilde{X}-t)\right) \\
\tilde{V}_{P 0 \tilde{\eta}}^{(1)}= \pm \pi^{-1 / 2} \int_{0}^{\infty} t^{-1 / 2}\left[F_{ \pm}(\tilde{X}-t)\right]_{Z} \exp \left(-\tilde{\eta}^{2} / 4 t\right) \mathrm{d} t \\
\tilde{W}_{0}^{(1)}=\mp(c / \pi \Delta)^{-1 / 2} \int_{0}^{\infty} t^{-1 / 2} F_{ \pm}(\tilde{X}-t) \exp \left(-\tilde{\eta}^{2} / 4 t\right) \mathrm{d} t
\end{gathered}
$$

As the meanflow correction is larger in the diffusion layer than the critical layer, the leading order nonlinear jump will result from the interaction of the meanflow and the largest fundamental in this (the diffusion) layer. 
(ii) The largest forced fundamental

The governing equations for the largest fundamental due to non-neutrality effects are

$$
\begin{gathered}
i \alpha \tilde{U}_{1}^{(2)}+(c / \Delta)^{1 / 2} \tilde{V}_{P 1 \tilde{\eta}}^{(2)}+\tilde{W}_{1 Z}^{(2)}=0, \\
i \alpha(\Delta / c)^{1 / 2} \lambda \tilde{\eta} \tilde{U}_{1}^{(2)}+\lambda \tilde{V}_{P 1}^{(2)}+(\Delta / c)^{1 / 2} \lambda_{Z} \tilde{\eta} \tilde{W}_{1}^{(2)}+i \alpha \tilde{P}_{1}^{(2)}= \\
-\tilde{U}_{0}^{(1)} \tilde{U}_{1 X}^{(1)}-(c / \Delta)^{1 / 2} \tilde{V}_{P 1}^{(1)} \tilde{U}_{0 \tilde{\eta}}^{(1)}-\tilde{W}_{1}^{(1)} \tilde{U}_{0 Z}^{(1)}, \\
\tilde{P}_{1 \tilde{\eta}}^{(2)}=0, \\
i \alpha(\Delta / c)^{1 / 2} \lambda \tilde{\eta} \tilde{W}_{1}^{(2)}+\frac{1}{\Delta} \tilde{P}_{1 Z}^{(2)}=-\tilde{U}_{0}^{(1)} \tilde{W}_{1 X}^{(1)} .
\end{gathered}
$$

These can be combined to give an equation for $\tilde{V}_{P 1}^{(2)}$ :

$$
\tilde{\eta} \tilde{V}_{P 1 \tilde{\eta} \tilde{\eta}}^{(2)}=(c / \Delta)^{1 / 2} \lambda^{-1} A(\tilde{X}) \frac{\partial}{\partial \tilde{\eta}}\left[-2 i \alpha d_{1} \tilde{\eta}^{-1}\left(\tilde{U}_{0 Z}^{(1)}-\frac{\lambda_{Z}}{\lambda} \tilde{U}_{0}^{(1)}\right)+d_{2} \tilde{U}_{0 \tilde{\eta}}^{(1)}\right]
$$

substituting for $\tilde{U}_{0}^{(1)}$ from equation (5.6) gives the required nonlinear jump

$$
\left[\tilde{V}_{P 1 \tilde{\eta}}^{(2)}\right]_{-\infty}^{\infty}=\frac{\Delta^{1 / 2}}{\lambda c^{3 / 2}} A(\tilde{X})\left(d_{2}-\frac{i \alpha d_{1}}{\Delta}\left(\partial_{Z}-\frac{\lambda_{Z}}{\lambda}\right) \Delta\right) \int_{0}^{\infty} \lambda^{2}\left(\frac{F_{w}(\tilde{X}-\zeta, Z)}{\lambda}\right)_{Z} \mathrm{~d} \zeta
$$

(iii) The evolution equation

Noting that $F_{w}$ is separable in $\tilde{X}$ and $Z$, we introduce $F_{w w}(Z)$ such that

$$
F_{w}(\tilde{X}, Z)=F_{w w}(Z)|A(\tilde{X})|^{2}
$$

Equation (5.8) may then be written in the form

$$
\left[\tilde{V}_{P 1 \tilde{\eta}}^{(2)}\right]_{-\infty}^{\infty}=\frac{\Delta^{1 / 2}}{\lambda c^{3 / 2}}\left(d_{2}-\frac{i \alpha d_{1}}{\Delta}\left(\partial_{Z}-\frac{\lambda_{Z}}{\lambda}\right) \Delta\right) \lambda^{2}\left(\frac{F_{w w}(Z)}{\lambda}\right)_{Z} A(\tilde{X}) \int_{0}^{\infty}|A(\tilde{X}-\zeta)|^{2} \mathrm{~d} \zeta .
$$

Thus the final evolution equation can be obtained from (3.15) and (5.9b). If the constants $\gamma_{1}, \gamma_{2}$ are defined by

$$
\gamma_{1}=\int_{0}^{2 \pi}\left\{f_{0}^{\infty} \frac{q R_{2}^{(0)}}{(\bar{u}-c)^{2}} d \xi-\frac{3 i \pi q_{10} \Delta \beta_{3 L}^{(0)}}{\lambda^{2} f^{2}}\right\} d Z / \int_{0}^{2 \pi}\left\{f_{0}^{\infty} \frac{q R_{2}^{(1)}}{(\bar{u}-c)^{2}} d \xi-\frac{3 i \pi q_{10} \Delta \beta_{3 L}^{(1)}}{\lambda^{2} f^{2}}\right\} d Z
$$




$$
\begin{aligned}
\gamma_{2}=\int_{0}^{2 \pi}\left\{\frac{i \alpha q_{10}}{c f \lambda^{2}}\left(d_{2}-\frac{i \alpha d_{1}}{\Delta}\left(\partial_{Z}-\frac{\lambda_{Z}}{\lambda}\right) \Delta\right) \lambda^{2}\left(\frac{F_{w w}(Z)}{\lambda}\right)_{Z}\right\} d Z / \\
\int_{0}^{2 \pi}\left\{f_{0}^{\infty} \frac{q R_{2}^{(1)}}{(\bar{u}-c)^{2}} d \xi-\frac{3 i \pi q_{10} \Delta \beta_{3 L}^{(1)}}{\lambda^{2} f^{2}}\right\} d Z,
\end{aligned}
$$

then the required evolution equation is

$$
\frac{d A}{d \tilde{X}}+\gamma_{1}\left(x_{0}\right) A=\sigma \gamma_{2}\left(x_{0}\right) A(\tilde{X}) \int_{0}^{\infty}|A(\tilde{X}-\zeta)|^{2} \mathrm{~d} \zeta, \quad A \rightarrow 0 \quad \text { as } \quad \tilde{X} \rightarrow-\infty
$$

Here the parameter

$$
\sigma=\frac{\epsilon^{2} R e^{2 / 3}}{\mu^{2}}
$$

measures the 'competitiveness' of the nonlinear term, in the evolution equation, relative to the term corresponding to linear effects. For $\sigma \ll 1$, linear effects will dominate the evolution and the disturbance will continue to grow after $x=x_{0}$. Here we concern ourselves with the regime $\sigma \sim O(1)$, where linear and nonlinear effects have equal influence on the evolution of the disturbance. A more general form of (5.11) can be derived which contains an additional 'linear' term, proportional to $\tilde{X} A$, to account for non-parallelism effects which are important for certain ranges of the parameters $(\epsilon, \mu, R e)$ cf Hall \& Smith (1984); Smith, Brown \& Brown (1993).

Thus we see that the $Z$ dependence of the problem has been removed by the application of the solvability condition so that the $Z$ dependence of the nonlinear problem is nonlocal as was found to be the case in the linear regime. Further, we see that our weakly nonlinear analysis has lead to a cubic nonlinearity; however rather than appearing as a polynomial (eg. as $A|A|^{2}$ if the evolution was described by the Stuart-Watson method), the nonlinear term is a convolution. The evolution equation is an integro-differential equation which depends on the entire history of the disturbance. Such evolution equations were first derived/proposed by Hickernell (1984). In fact Wu (1993) and Smith, Brown \& Brown (1993) have derived essentially the same equation in their studies of boundary-layer transition; however, as we have considered a fully three-dimensional boundary-layer, our coefficients $\gamma_{1}, \gamma_{2}$ are far more complicated. Similar equations have also appeared in other recent papers eg. Smith \& Walton (1989), Smith \& Blennerhassett (1992), Wu, Lee \& Cowley (1993) and Blackaby (1994).

\section{Solution of the evolution equation and conclusions}

The nature of the solution of (5.11) depends crucially on the sign of the real part of $\gamma_{2}$ (defined by equation (5.10b). Since the disturbance under investigation becomes unstable as it moves downstream we know that the real part of $\gamma_{1}$ is positive. Without calculating the solution of the neutral leading order eigenfunction and adjoint problems we cannot say what is the sign of 
the real part of $\gamma_{2}$. We shall therefore discuss both possibilities and use results from experiments to suggest the most likely scenario. Firstly we note that a suitably rescaled version of (5.10) takes the form:

$$
\frac{d B}{d \bar{X}}=B \pm B(\bar{X}) \int_{0}^{\infty} B(\bar{X}-\zeta) \mathrm{d} \zeta, \quad B \rightarrow 0 \quad \text { as } \quad \bar{X} \rightarrow-\infty
$$

Here $B$ is real, positive and proportional to $|A|^{2}$ and the \pm signs correspond respectively to the cases when the real part of $\gamma_{2}$ is positive and negative respectively.

The solution of (6.1) which has the required upstream behaviour is given by

$$
B=\frac{2 e^{\bar{X}}}{\left(1 \mp e^{\bar{X}}\right)^{2}} .
$$

If we take the negative sign in (6.2), corresponding to the positive sign in equation (6.1), we see that a singularity develops after a finite distance. If the positive sign is taken in (6.2), $B$ grows as $\bar{X}$ increases from $-\infty$ until it reaches a maximum and then decays exponentially to zero. At first sight this seems a rather curious fate for a disturbance which was initially unstable on the basis of linear theory. However the integral term in (6.1) can be interpreted as the effect on the growth rate by the mean flow corrected by the upstream development of the instability. Thus the mean flow modification which occurs in the early stages of the growth of the disturbance adjusts the meanflow so as to make it linearly stable further downstream.

Experimental observations certainly suggest that the mode identified by Hall and Horseman (1991) continues to grow after it first becomes unstable. Here we are assuming that the mode of instability discussed by Hall and Horseman (1991) is responsible for the experimentally observed onset of three-dimensionality in the Görtler problem. The closeness of the theoretically predicted most unstable wavenumber and frequency with those measured by Swearingen and Blackwelder (1987) gives some backing for that assumption. The experiments therefore suggest that the negative sign is appropriate in (6.2). However without numerical solutions of the neutral eigenfunction and its adjoint we cannot confirm that assertion.

We have carried out a viscous critical layer analysis for a marginally unstable inviscid disturbance to a flow containing a streamwise vortex structure. The vortex structure could be the result of a centrifugal instability, wave interactions or other mechanisms. In fact our analysis is valid for any flow where one of the velocity components depends on two spatial variables and is larger than the the other two components. For such more general flows, the periodicity in the spanwise direction, which we assume in this paper, must be replaced by an appropriate condition in order to derive the required solvability condition.

Our analysis is similar to that of Wu (1993) and Smith, Brown \& Brown (1993); however our analysis is complicated due to the fact that the nonlinear vortex state has rendered the boundary-layer flow three-dimensional. As a consequence, our disturbances have a general $Z-$ dependence whereas Wu (1993) was able to consider separate harmonics in $Z$ and derives coupled amplitude equations. In the problem considered by Smith, Brown \& Brown (1993), the initial boundary-layer flow is two-dimensional; all subsequent vortex activity (three-dimensionality of the boundary-layer) is due to the relatively large mean-flow corrections induced in the diffusion (buffer) layers via nonlinear-interaction effects. 
In this paper we have described the evolution of the inviscid modes found by Hall and Horseman (1991); this has done using viscous-critical-layer and diffusion-layer theories in the context of a weakly nonlinear instability theory. In particular, we have considered the evolution of a mode near the critical streamwise location where the vortex structure has developed sufficiently to (first) render the now three-dimensional boundary-layer flow unstable to inviscid modes. At such a location, the flow is marginally unstable and we can consider the evolution of the most dangerous (important) mode. We note that our theory is not directly applicable to modes excited at streamwise locations where the flow supports a band of unstable modes (i.e. at an $\mathrm{O}(1)$ distance downstream from the critical $x$-location); in such cases the most dangerous mode has too large a growth rate and the wavenumber will not be close enough to a "neutral' value for weakly-nonlinear theory to be immediately applicable. However, it can be argued that viscous spreading effects (or some other external effect) will reduce the growth rates to a size where a weakly nonlinear theory (based on unsteady critical-layer theory rather than viscous critical-layer theory) is appropriate. The papers by, for example, Michalke (1964); Crighton \& Gaster (1976) and Hultgren (1992) support such an argument, which has been used in many recent papers concerned with flow stability eg. Goldstein \& Leib (1988); Goldstein \& Hultgren (1988); Goldstein \& Leib (1989); Hultgren (1992); Wu, Lee \& Cowley (1993). The evolution of the Hall-Horseman-modes for the non-marginal stability case is the subject of current study by the authors and will be reported on in due course.

Our analysis shows that the disturbance amplitude satisfies the integro-differential equation (5.11/6.1). Experimental observations show that the linear growth of three-dimensional disturbances to Görtler vortices is rapidly followed by the onset of turbulence. Such a scenario would be consistent with $(6.1)$ if the positive sign were taken in that equation. The excellent agreement between the experimental measurements of Swearingen and Blackwelder (1987) and Hall and Horseman (1991) for the linear regime lead us to believe that this is indeed the case. However it is possible that the sign to be taken in $(6.1,2)$ depends on the wavenumber and frequency of the marginally unstable mode and that some disturbances are destroyed by viscous effects. Therefore it is conceivable that the linearly growing disturbances are inhibited by viscosity for weak vortex states and grow explosively further downstream when the vortex state has been reinforced.

Following the explosive growth of the disturbance new effects must come into play and viscosity will play a secondary role; see Wu, Lee and Cowley (1993) who were concerned with the growth of inviscid disturbances to Stokes layers. The extension of our work along the line followed by the latter and indeed other authors is made nontrivial by the fact that in our calculation the critical layer is not flat. 


\section{References}

Blackaby, N.D. (1994), Tollmien-Schlichting/vortex interactions in compressible boundary layer flows. IMA Journal of Applied Math, in press.

Brown, P.G., Brown, S.N., Smith, F.T. \& Timoshin, S.N. (1993), On the starting process of strongly nonlinear vortex/Rayleigh-wave interactions. Mathematika 40, 7-29.

Brown, S.N. \& Stewartson, K. (1978) The evolution of the critical layer of a Rossby wave. Part II. Geophys. Astrophys. Fluid Dyn. 10, 1-24.

Crighton, D. G. \& Gaster, M. (1976) Stability of slowly divergent jet flows. J. Fluid Mech. 77, $397-413$.

Goldstein, M. E. \& Hultgren, L. S., (1988), Nonlinear spatial evolution of an externally excited instability wave in a shear layer. J. Fluid Mech. 197, 295-330.

Goldstein, M. E. \& Leib, S. J., (1988), Nonlinear roll-up of externally excited free shear layers. J. Fluid Mech. 191, 481-515.

Goldstein, M. E. \& Leib, S. J., (1989), Nonlinear evolution of oblique waves on compressible shear layers. J. Fluid Mech. 20\%, 73-96.

Hall, P. (1988), The nonlinear development of Görtler vortices in growing boundary layers. J. Fluid Mech. 193, 243-266.

Hall, P. \& Bennett, J. (1986) Taylor-Görtler instabilities of Tollmien-Schlichting waves and other flows governed by the interactive boundary layer equations. J. Fluid Mech. 171, 441-458.

Hall, P. \& Horseman, N.J. (1991), The linear inviscid secondary instability of longitudinal vortex structures in boundary layers. J. Fluid Mech. 232, 357-375.

Hall, P. \& Lakin, W.D. (1988), The fully nonlinear development of Görtler vortices in growing boundary layers. Proc. R. Soc. Lond. A 415, 421-444.

Hall, P. \& Seddougui, S. O. (1989), On the onset of three-dimensionality and time-dependence in the Görtler vortex problem. J. Fluid Mech. 204, 405-

Hall, P. \& Smith, F.T. (1991), On strongly nonlinear vortex/wave interactions in boundary-layer transition. J. Fluid Mech. 22\%, 641-666.

Hickernell, F.J. (1984), Time-dependent critical layers in shear flows on the beta-plane. J. Fluid Mech. 142, 431-449. 
Hultgren, L. S. (1992). Nonlinear spatial equilibration of an externally excited instability wave in free shear layer. J. Fluid Mech. 236, 635-664.

Li, F. \& Malik, M. (1994) On the breakdown of Görtler vortices: Nonlinear Development and Secondary Instabilities. submitted for publication.

Liu, W. \& Domardski, J. A. (1993), Direct Numerical Simulation of Transition to Turbulence in Görtler Flow. J. Fluid Mech. 246 267-299.

Michalke, A. (1964) On the inviscid instability of the hyperbolic tangent velocity profile. J. Fluid Mech.. 233, 543-556.

Smith, F.T. \& Blennerhassett, P.J. (1992), Nonlinear interaction of oblique three-dimensional Tollmien-Schlichting waves and longitudinal vortices, in channel flows and boundary layers. Proc. R. Soc. Lond. A 436, 585-602.

Smith, F.T., Brown, S.N. \& Brown, P.G. (1993) Initiation of three-dimensional nonlinear transition paths from an inflectional profile. Eur. J. Mech. B/Fluids 12, 44\%-473.

Smith, F.T. \& Walton, A.G. (1989) Nonlinear interaction of near-planar TS waves and longitudinal vortices in boundary-layer transition. Mathematika 36, 262-289.

Swearingen, J.D. \& Blackwelder, R.F. (1987) The growth and breakdown of streamwise vortices in the presence of a wall. J. Fluid Mech. 182, 255-290.

Wu, X. (1993) On critical-layer and diffusion-layer nonlinearity in the three-dimensional stage of boundary-layer transition. Proc. R. Soc. Lond. A 443, 95-106.

Wu, X., Lee, S.S. \& Cowley, S.J. (1993) On the weakly nonlinear three-dimensional instability of shear layers to pairs of oblique waves - the stokes layer as a paradigm. J. Fluid Mech. 253, 681-721.

Yu, X. \& Liu, J. T. C. (1994) On the mechanism of sinuous and varicose modes in threedimensional viscous secondary instability of Görtler rolls. Physics of Fluids, 6, 2, 724-735. 


\section{Figure Captions}

Figure 1. The growth rates of the two most dangerous odd modes at a position $100 \mathrm{~cm}$ from the leading edge in the experiment of Swearingen and Blackwelder (1987).

Figure 2. The contours of the streamwise and normal velocity components for the most dangerous odd mode $100 \mathrm{~cm}$ from the leading edge in the experiment of Swearingen and Blackwelder (1987).

Figure 3. The various regions of the three-dimensional boundary-layer flow: Ia,b The outer flow; IIa,b The diffusion layers of relative thickness $O\left(R e^{-1 / 4} \mu^{-1 / 2}\right)$; III Critical layer of relative thickness $O\left(R e^{-1 / 6}\right)$; IV Outer wall layer of relative thickness $O\left(R e^{-1 / 6}\right)$; V Inner wall layer of relative thickness $O\left(R e^{-1 / 4}\right)$. Note that (i), these thicknesses are given relative to the boundary-

layer thickness $O\left(R e^{-1 / 2}\right)$, and (ii), the critical and diffusion layers are distinct from the wall being centered around the level $Y=f(x, Z)$. 